\title{
Integration in Operation Theater: Need of the Hour
}

\author{
${ }^{1}$ Vijaydeep Siddharth, ${ }^{2} \mathrm{~S}$ Kant, ${ }^{3} \mathrm{R}$ Chandrashekhar, ${ }^{4}$ Shakti Kumar Gupta
}

\begin{abstract}
Integration in operation theater (OT) usually refers to systems integration, which means functionally connecting the OT environment. It includes integration of patient information system, audio, video, surgical lights and room lights, building automation (HVAC), medical equipment, telemedicine, videoconferencing, etc. When integrated, all technology can be manipulated from a central command console by single operator. Integration in OT holds the key for effective application of minimally invasive surgery, robotic surgery and functioning of hybrid OT. Apart from the conventional planning team for an OT, bringing on board an IT professional is a must for planning IT requirements. Integration in OT requires $15 \%$ more space than the conventional OT in order to accommodate the IT gadgets, i.e. walk-in technology room that would house teleconferencing and $\mathrm{A} / \mathrm{V}$ equipment, blade-server computers, fiberoptic network electronics, teleconferencing and video conferencing equipment, audio-visual racks, etc. the level of integration can vary within an OT depending upon the user requirement. Space requirement for a general I-OT is about $60 \mathrm{~m}^{2}$, a cardiovascular I-OT or one that includes robotics may come up to $80 \mathrm{~m}^{2}$ or more, and I-OT with in-room imaging equipment requires at least $100 \mathrm{~m}^{2}$. Integration in OT not only increases the efficiency and patient safety in delivery of healthcare but can significantly increase efficiency and effectiveness both in teaching and research.
\end{abstract}

Keywords: Integrated OT, Operation theater, Planning and designing, Surgery.

How to cite this article: Siddharth V, Kant S, ChandrashekharR, Gupta SK. Integration in Operation Theater: Need of the Hour. Int J Res Foundation Hosp Healthc Adm 2015;3(2):123-128.

Source of support: Nil

Conflict of interest: None

\footnotetext{
${ }^{1}$ Assistant Professor, ${ }^{2}$ DDG (OPP) ${ }^{3}$ Advisor (Architecture)

${ }^{4}$ Medical Superintendent

${ }^{1}$ Department of Hospital Administration, All India Institute of Medical Sciences, New Delhi, India

${ }^{2}$ Office of DGMS (Army), Ministry of Defence, Government of India, New Delhi, India

${ }^{3} \mathrm{HLL}$ Lifecare Ltd. (A Government of India Enterprise); London South Bank University and Ministry of Health and Family Welfare, Government of India, New Delhi, India

${ }^{4}$ All India Institute of Medical Sciences, Dr RP Centre for Ophthalmic, New Delhi, India
}

Corresponding Author: Vijaydeep Siddharth, Assistant Professor, Department of Hospital Administration, Room No. 6A, All India Institute of Medical Sciences, Ansari Nagar, New Delhi-110029, India, Phone: 9013844255 , e-mail: dr.siddharth mamc@gmail.com

\section{INTRODUCTION}

An operation theater (OT) complex is the 'heart' of any hospital/surgical center. All the skills of surgical professional and new scientific developments will be of little value unless the operating suite is properly planned and works efficiently. ${ }^{1}$ The rapid growth of minimally invasive surgery (MIS) and also because of the ergonomically poor design of traditional operating rooms which involved a number of carts to be mobilized at the time of surgery has compeled significant developments in OT planning and designing. Not only this, digital information has become the standard format and universal way of accessing patient information and communicating with healthcare personnel. The OT requires easy real time access to these digital data and a way of managing the digital information acquired within the OT. More complex procedures in the interventional suite mean patients will require more clinical supervision and possibly a more intense level of care. This has been an important factor in shift from conventional OT toward integrated OTs. There is immense requirement for renovation of traditionally designed OTs in hospitals to facilitate the practice of MIS. ${ }^{2}$

\section{WHAT DO WE MEAN BY INTEGRATION IN OT?}

Integration in planning and design of OT can be seen from perspective of service integration and systems integration. In service integration, single authorized vendor is responsible for getting all kinds of the work done along with coordination with all other service providers. But, conventionally integration in OT is referred to systems integration, which means functionally connecting the OT environment. It includes integration of patient information system, audio, video, surgical lights and room lights, building automation (HVAC), medical equipment, etc. (Fig. 1). Healthcare personnel can easily route audio visual sources and effectively control surgical equipment. When integrated, all technology can be manipulated from a central command console by single operator.

When compared against a nonintegrated OT, where an assortment of equipment is arranged around the surgical table and individual pieces are pulled up or pushed back as and when needed. A circulating nurse works amidst the equipment to change settings during the surgery/ procedure. Cables and cords from the equipment lie in 


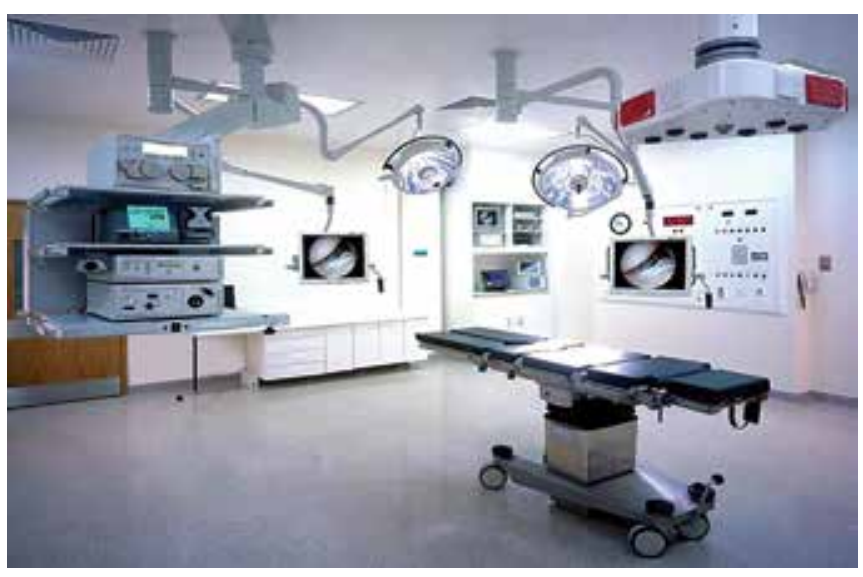

Fig. 1: Integrated OT

the path of the nurse, the patient gurney, even under the feet of the anesthesiologist and the surgical team. There is an inherent risk of tripping and disturbing the surgery, of pulling out wires, and of damaging equipment in such kind of setting. Figure 2 clearly portrays the difference that is brought to OT once it has been converted into an integrated OT.

In an integrated OT, cords and cables run inside the articulating arms of ceiling-mounted lights and booms through conduits in the ceiling. Ideally, there are no cords or cables on the floor. The circulating nurse works from a console at a control station, where she has access via a computer to numerous devices in the OT and no longer keeps navigating during the surgery. In fact, she is not even accessing the sterile area. All controls are on a touch screen, and the nurse can carry out the surgeon's requests from this common control station for all equipment. A surgeon may also have a command console within reach in the surgical field. Some systems are capable of even offering voice recognition, so that the surgeon wearing a wireless headset with a microphone can control the system directly. This not only results in lesser chaos inside a stressful setting but also reduces the movement and number of personnel entering the sterile area. In teaching hospitals where the surgical procedures are to be demonstrated to the medical graduates and postgraduates, an integration of IT technology not only provides better understanding and visibility through high definition displays but also reduces the number of personnel within the OT. This indirectly have an impact on infection control within the OT.

\section{BENEFITS OF INTEGRATED OT ${ }^{3}$}

An integrated OT means saving time and personnel because more procedures can be completed in the same room by the existing staff without increasing the strain on the team and without relocating equipment or personnel from another OT. ${ }^{4}$

- Hospitals are increasingly seeking to build stateof-the-art OTs so as to attract and retain healthcare personnel by offering them the advanced facility.

- There is overall improvement in the efficiency and patient safety inside the OT, having integrated technology, e.g. knowledge or bulletin board systems are now available that integrate patient and staff information as well as equipment and process documentation, and post them on a wide screen in the room. A preincision screen, intraoperative screen, closing screen, and case-summary screen bring together an arrangement of real-time data, such as the patient's vital signs, blood loss, and fluid levels. And because simple human errors, such as misidentification of a patient, can lead to lifethreatening medical errors, the knowledge/bulletin board displays the procedure name, patient name, gender, age, weight, and critical information, such as allergies and special needs. In addition, the names of all staff in the OT are listed. The goal is to manage and coordinate details in order to reduce medical errors.

- Design for technology integration improves the quality of data captured and workflow. The surgeon looks comfortably at his or her screens as opposed to

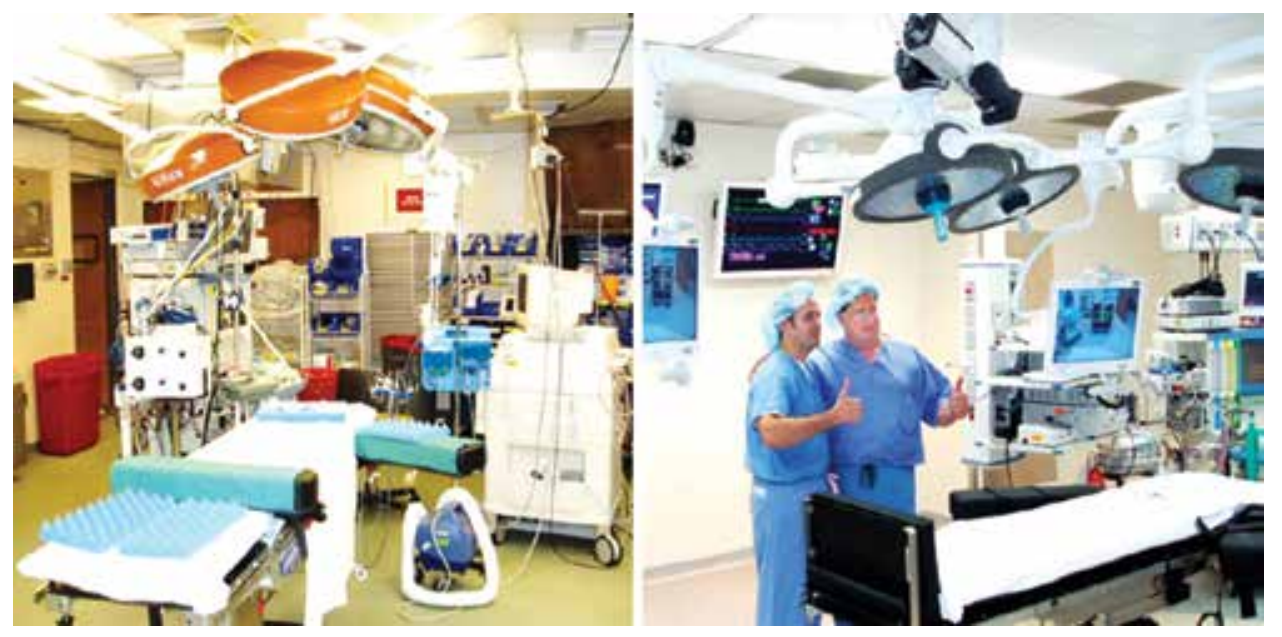

Fig. 2: Operation theater before and after integration 
looking to the side or behind to a system-on-a-cart solution. This has not only improved patient safety but also added to safety of staff by having better ergonomics of OT.

- Repetitive suturing and other surgical tasks can cause hand, back and leg fatigue. This requires frequent resting, extended surgery time, and varying degrees of precision. Integrated systems coupled with MIS minimize surgeon fatigue and improve patient outcomes. Many surgeons have a calming and moodsetting music playlist designed for their process. Not unusual are music selections chosen for opening, focus, and a closing celebration.

- With increased control of OT environment, the surgical team can work more efficiently and reduce operating time.

- Integrating various OT components into one centrally controlled system results in optimized processes.

- Setup and clean-up are faster and easier because equipment does not have to be connected and disconnected between procedures. The appropriate equipment swings into place via overhead booms, and monitors are easily positioned for optimal viewing by each surgical team.

- Unprecedented operating efficiency correlates with increased productivity and better utilization of every OT every day.

- The ability to reconfigure a room by using the control console to select the case type or surgeon setup makes the rooms flexible. The intent is to enable the facility to adapt to future additions, changes, and use/practice of technology.

- It also brings effectiveness and efficiency in training and research.

\section{PLANNING FOR INTEGRATED OT}

When an OT is being built or renovated, incorporating I-OT has a significant impact on planning and design. In this article, only the additional planning and design parameters of integration required in a conventional OT are discussed. The other planning and design parameters for, e.g. HVAC, lighting, infection control, space requirements for ancillary areas, etc. will be same like any other OT. It is important not only to consider what is needed now but also to plan ahead for what will be needed in the foreseeable future. Some picture archival and communication system (PACS) are centrally located miles away from the hospital. File sizes of the images to support intervention may be extremely large-from 50 to $900 \mathrm{MB}$. Over conventional networks, it might take half an hour for a server to get these images to the I-OT. Therefore, the IT process flow for PACS and the network need early evaluation and planning.

\section{Planning Team}

A qualified medical technology planning firm should be brought on board at the earliest planning stage to coordinate with all stakeholders. Planning team should consist of following members: ${ }^{5}$

- Hospital administrator

- Anesthetist

- Surgeons

- Clinicians

- Hospital infection control personnel

- IT department

- Radiology

- Biomedical engineering

- Architects

- Contractor

- Other stakeholders.

To help the clinical/physician staff reach decisions on the technology, the technology planner should coordinate with a variety of vendors to conduct on-site equipment evaluations. Also, the planner should provide a detailed work plan and guidance on integrating radiology, cardiology, gastroenterology, orthopedics, anesthesiology, and nursing and physicians.

\section{Design Considerations}

Integrated OT, when optimally designed, can lessen the complexity of the most complicated environment in the hospital-the surgery suite. User-friendly, integrated technologies augment surgeons' skills and help the entire surgical teamwork more safely and efficiently. The development and growth of MIS has spurred the creation of I-ORs. Today, more than half the surgery cases in the United States are performed with minimally invasive techniques.

A high-quality, magnified video, projected on a flatscreen monitor, gives the surgeon an optimal view of the surgical field. As minimally invasive surgery continues to grow and robotic surgery and telemedicine become more common, I-OTs will become an industry standard. ${ }^{5}$

\section{Levels of Integration ${ }^{5}$}

There are various levels to which an integrated OT can be made according to user requirement. The following classification of integrated OT is based on the basis of communication of I-OT with internal and external environment of the OT complex/department:

Level 1 integration: Level 1 integration, refers to where only internal environment of the OT is integrated, i.e. where audio, video, lighting, HVAC, music, within the OT is integrated. 
Level 2 integration: In level 2 integration, OT is connected/ integrated with other OT within the OT complex. By getting this level of integration, it is possible to video conference with other OT and take expert opinion while operating in other room. This brings in effectiveness and improves the quality of care rendered to patients. In this way, it also helps in capacity development of staff and on the job training.

Level 3 integration: In integrated OT of level 3, OT is not only capable of communicating within the OT complex, seminar room or classroom within the same hospital but can communicate with facilities located across the countries, continents with the help of ISDN line.

Another classification which is being followed depending upon the integration of different technologies and level of system integration:

$V E$ level: At the minimal, or VE level, video from medical equipment is fixed in the room. The video sources are images from the arthroscopic, laparoscopic, and endoscopic cameras. These video images are displayed on specific flat-panel monitors hung from the ceiling boom or on a cart near the surgeon.

AVT level: Classic $\mathrm{A} / \mathrm{V}$ and teleconferencing systems are at the AVT level of integration. These systems are used for conference room and auditorium presentations and may have music, television, and projection systems.

AVER level: The next level of integration is the AVER level (audio, video, medical equipment, room environment), which enables easy access and control of all equipment and video switching. Video switching, or routing, allows for video images to be redirected to two or more displays. These audio systems, surgical video systems, and room environment controls are interlinked so that the surgeon (via a nurse at the central control unit or via voice activation) can manipulate a multitude of devicesfor example, an in-surgical-light camera, a surgical instrument light source, a vacuum pump, room lights and temperature, and video conferencing. All equipment is integrated and working together.

AVERPI level: In general, when we speak of I-OTs, we are referring to at least an AVERPI level of integration (audio, video, medical equipment, room environment, Picture Archive and Communications System (PACS), and information system). At this level, the I-OT is connected to other areas of the facility, such as radiology and laboratories. PACS images-computer desktop images with data images, such as lab results-can be routed to displays within the I-OT suite and digital picture images taken earlier can be accessed and viewed on monitors. A specialized information system may collect information specific to the case and present it on a large display at the wall to organize the surgery and its functional progress. This display of case knowledge improves safety while documenting the progress and maintaining staff orientation. When the surgeon takes a biopsy and sends it to the lab for analysis, images from the lab microscope can be sent back to the I-OT so that the surgeon and the pathologist can consult via phone while the surgery is in progress, avoiding delays that in the past would have meant a second surgery.

AVERPIT level: The most fully integrated systems, at the AVERPIT level, add telemedicine, which connects the I-OT to the outside world. Audio/video conferencing extends communication abilities to conference rooms and classrooms across the street or around the world, so that surgeons can consult and teach remotely. In fact, this level extends the skill set of a specialty surgeon, thus helping to expand a scarce resource.

At the AVERPIT, or highest, level of integration, audio, video, medical equipment, room environment, PACS, information systems and telecommunications all work together in the OT. Images and data can be resourced or shared with other areas of the hospital, and tele/ videoconferencing capabilities extend to the outside world (Fig. 3).

The AVERPI and AVERPIT levels of integration provide the flexibility to utilize the surgical suite for many types of cases, which is advantageous for the hospital. However, large capital allocations are required for these levels of OT integration. Therefore, it is recommended to design and build all OTs to be adaptable for accommodating these levels of technology integration in the future. Otherwise, the OT would have to be shut down for wall and ceiling work, creating infectioncontrol issues and lost revenue during construction. The 'adaptable' approach may be compared to the 'universal' approach which requires all OTs to be constructed for all levels and be fully fitted-out with equipment.

\section{Design Features for Integrated OT}

In addition to housing a large amount of technology, I-OTs impact work flow. Factors that must be considered in the design of an I-OT include:

- Total room size

- Best placement of lights, cameras, monitors, and the $\mathrm{AV}$ equipment

- Ceiling structures

- Articulating display arms

- Equipment booms should be designed to allow for added capabilities in the future.

Additional space is required for the infrastructure and electronics to support the components outside the OT. 


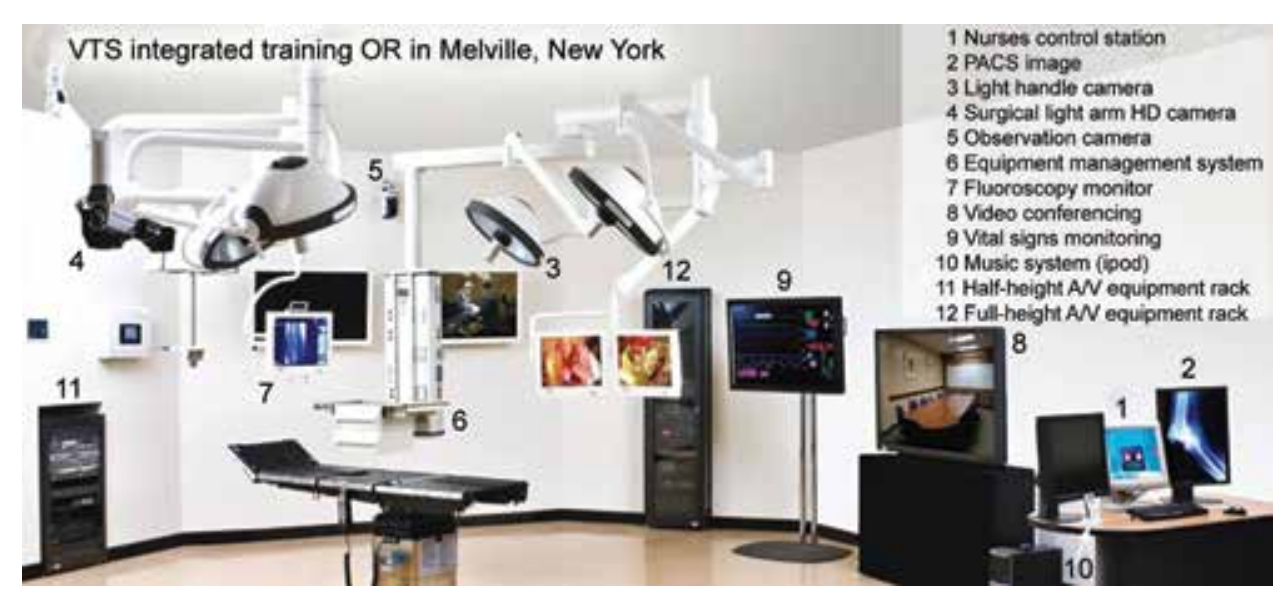

Fig. 3: Integrated training OT in Melville, New York $^{3}$

\section{Room Size}

In general, I-OTs are $15 \%$ larger than their nonintegrated counterparts. It is true that some equipment is smaller than ever before, but an in-room control or documentation center adds about $10 \mathrm{~m}^{2}$ to an OT. With integrated technology, a general I-OT is about $60 \mathrm{~m}^{2}$, a cardiovascular I-OT or one that includes robotics may approach $80 \mathrm{~m}^{2}$ or more, and I-OT with in-room imaging equipment requires at least $100 \mathrm{~m}^{2}$.

\section{Media Bridge}

Media bridge maximizes the quality of surgical interventions providing surgeons, anesthetists and OT team's easy access to power supplies, manifold supplies, communication technologies and data transfers that can be easily positioned and customized or moved as per the requirement for different surgical procedures. They can be customized as per user specifications in a configuration, with one, two or four corners in order to match the size and organization of space (Fig. 4).

They are compatible with systems of ceiling laminar airflow, the beams are suspended in such a manner so that they do not disturb the laminar flow and generate turbulence, thereby aiding in maintaining the aseptic environment of the OT. It also allows the positioning of the equipment trolleys as per the need and can be moved from one end to the other. Support trolleys may be positioned on the entire periphery of the beam through swivelling angles.

\section{Technology Room}

The I-OT support hardware has invaded the telecommunications closet. This closet should be of atleast $10^{\prime}$ $\times 15^{\prime}$ walk-in technology room that would house teleconferencing and $\mathrm{A} / \mathrm{V}$ equipment, blade-server computers in racks, fiberoptic network electronics, and fiberoptic cables to the work area. This has challenged the traditional structured cable system (SCS) design, in which fiber is used to move data vertically in a building and copper is used to move data horizontally. Fiber to the desktop is a reality for the interventional I-OT. Data transport via a $\mathrm{T} 1$ line, an integrated services digital network (ISDN), and a digital subscriber line (DSL) are common requirements for telemedicine data communications to the world.

The I-OT's video-integration technology often requires $3^{\prime} \times 3^{\prime} \times 6^{\prime}$ rack. In addition, there may be three or more additional computers to support PACS, hospital information data, electronic medical records, and/or care plan documentation. There is always a concern on where to place the rack, A/V electronics, and computers so they are not in the way during procedures or cleaning. One innovative solution is to create a dedicated closet for the rack. The closet has a door from the corridor and a door at the inside of the room. If the video equipment needs to be updated, changed, or serviced, it can be accessed from the corridor. The inside remains closed off so the I-OT can be used for procedures not requiring integration. Servicing the technology does not shut down the OT, and cleaning the OT does not disturb the equipment. The closet can be locked to secure the expensive commercial equipment.

In the surgery department outside the I-OTs, a department control desk with closed circuit TV technology and a large screen can facilitate efficiency in the suites. An image of every I-OT appears on the screen so that the viewer instantly knows the room statuswhether or not a procedure is in progress, if the room is vacant, clean or in need of cleaning, or if it is ready for the next procedure. Providing this feature is a great aid in efficient scheduling.

\section{CONCLUSION}

The integration in OT is a technology to reckon considering the rapid development of minimally invasive 


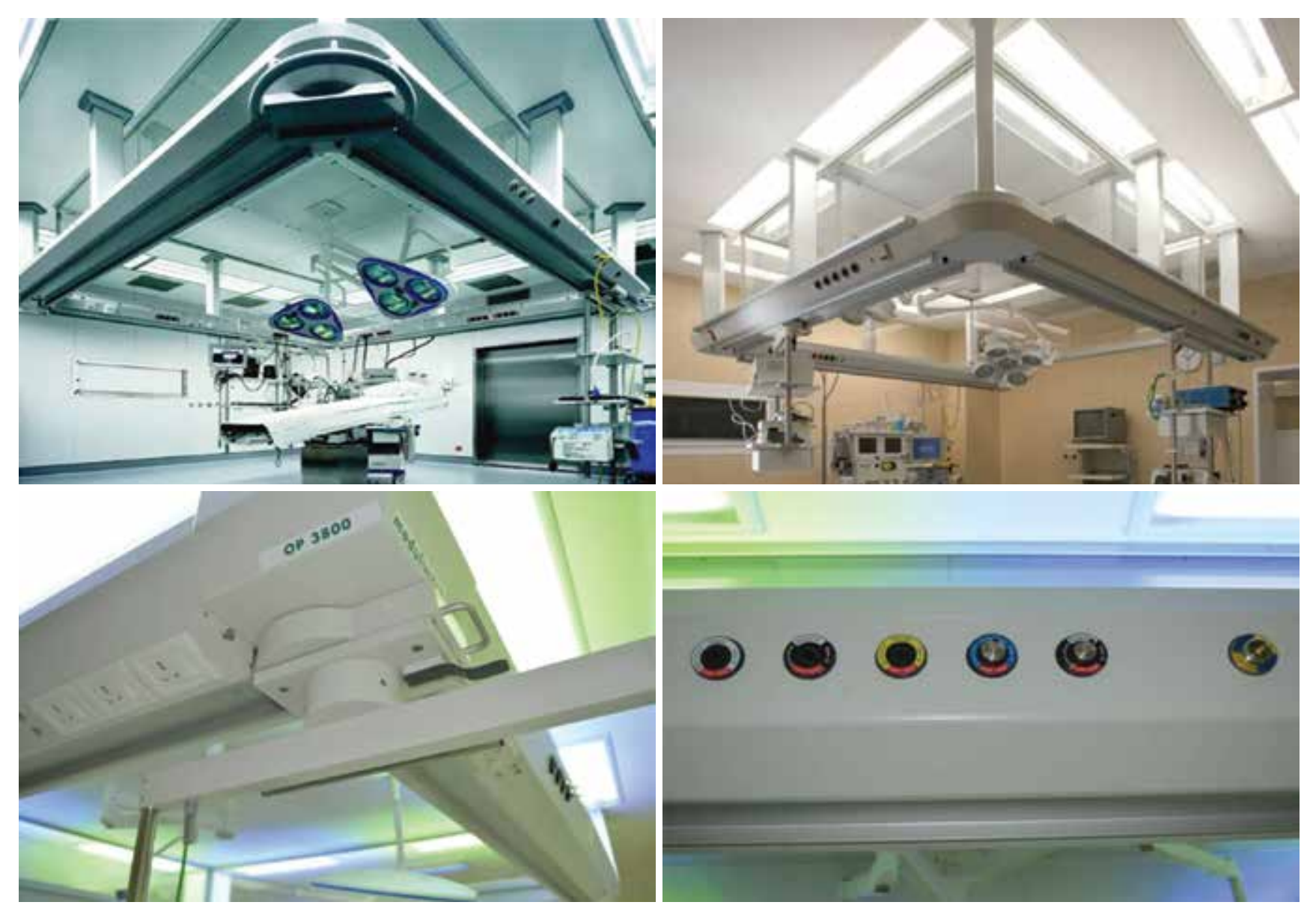

Fig. 4: Media bridge of an integrated OT (showing its various components)

surgery and robotic surgery. Also, the development of hybrid OT in hospitals has necessitated the integration in OT for its smooth functioning. In fact to the extent that all the OTs in a new or upcoming facility irrespective of the fact whether they are traditional/hybrid OT should be planned keeping the integration in mind. Integration in OT not only brings efficiency and patient safety in delivery of healthcare but can significantly increase efficiency and effectiveness both in teaching and research.

\section{REFERENCES}

1. Towers M. The modern conception of an operating theater suite. South African Med J [Internet] 1967 Feb 25;41(8):181-186. Available at: http://www.ncbi.nlm.nih.gov/ pubmed/6024606.
2. Stanbridge DD, Fried GM. Current developments in operating room design [Internet]. Operating Room 2007 p. 8-9. Available at: http://www.touchbriefings.com/pdf/2742/fried.pdf.

3. Systems Integration-Operating Room [Internet]. VTS Medical Systems [cited 2012 Oct 15]. p. 2. Available at: http://www. vtsmedical.com/pdf/datasheets/SystemsIntORrevB.pdf.

4. Kpodonu J, Raney A. The cardiovascular hybrid room a key component for hybrid interventions and image guided surgery in the emerging specialty of cardiovascular hybrid surgery. Interactive cardiovascular and thoracic surgery [Internet]. 2009 Oct [cited 2012 Oct 15];9(4):688-692. Available at: http://www.ncbi.nlm.nih.gov/pubmed/19622541.

5. Juett $S, \mathrm{O}^{\prime}$ Connor P. The Integrated operating room [Internet]. Healthcare Design Magazine. 2006 [cited 2012 Oct 16]. Available at: http://www.healthcaredesignmagazine.com/ article/integrated-operating-room. 